\title{
Static-static-light baryonic potentials
}

\author{
Johannes Najjar*, Gunnar Bali \\ Institut für Theoretische Physik, Universität Regensburg \\ 93040 Regensburg, Germany \\ E-mail: \\ johannes.najjar@physik • uni-regensburg.de, \\ gunnar.galiephysik.uni-regensburg.de
}

We determine doubly heavy baryonic potentials as a function of the distance between the two static sources, coupled to a light relativistic quark, for different quantum numbers. We use the variational method to compute the ground state and the first two excitations. These can be used as an input to nonrelativistic models or to NRQCD calculations of properties of doubly heavy baryons. We compare our findings with a factorization model. We employ all-to-all propagator methods, improved by an additional hopping parameter expansion and Wuppertal smearing on $N_{f}=2$ QCDSF configurations.

The XXVII International Symposium on Lattice Field Theory

July 26-31, 2009

Peking University, Beijing, China

${ }^{*}$ Speaker. 


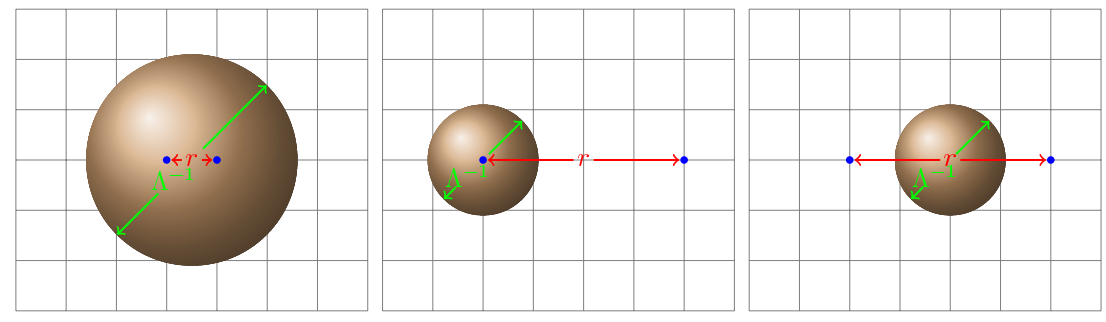

Figure 1: The different models for the static-static-light baryon.

\section{Introduction}

The coupling of quarks inside a doubly heavy baryon is debated and there are various models on the market like $\Delta$ - or $Y$-flux tubes between all three quarks or the formation of a diquark which couples to the third quark. The ground state doubly heavy baryon potential has been studied previously on the lattice [1]. Ref. [2] suggests that a diquark formation of the two heavy quarks is dynamically favored and that the light quark perceives this as an almost pointlike object. Therefore a $Q Q q$ baryon with two heavy quarks is related to a heavy-light $\bar{Q} q$ meson [3]. In the static limit where the spin of the heavy quark completely decouples, the main difference between these two systems is that the $Q Q$ diquark is spatially extended. In this work we check up to what $Q Q$ separations this scenario holds. In figure 1 we depict different situations. We ascribe a phenomenological Compton wavelength $\Lambda \sim 1 / m_{q}$ to a light quark of mass $m_{q}$. For $r \ll \Lambda^{-1}$ the light quark cannot resolve the two static quarks (left picture). When either the light quark mass or the static quark distance $r$ is increased, the light quark can discriminate between the static color sources and can "choose" its preferred localization. Symmetry considerations suggest the scenarios where the light quark sits on top of one of the static quarks or in the middle between them to be of particular interest. The first case corresponds to $Q q$ diquark formation while in the second case no diquarks are formed. Unlike in the physical situation of finite heavy quark masses where the average distance $\langle r\rangle$ is dynamically determined, in the static limit we can change $r$ to interpolate between the $Q Q$ diquark picture at small $r$ and the other more involved scenarios. At $r=0$ the spectra of the $Q Q q$ and $\bar{Q} q$ situations are (numerically and analytically) identical. As $r$ is increased the energies will increase. For instance, $V_{Q Q}=V_{\bar{Q} Q} / 2$, up to a constant, at least to the first two orders of perturbation theory and in the string picture. We denote mass levels of the mesonic $\bar{Q} q$ system by $m_{\bar{Q} q}$. Thus, in the $Q Q$-diquark picture, that at least should hold at $r<\Lambda^{-1}$, we expect the factorization,

$$
V_{Q Q q}(r) \approx m_{\bar{Q} q}+\frac{1}{2} V_{\bar{Q} Q}(r),
$$

which can graphically be depicted as,

$$
\exp \left(-\left.\{\xi) \propto \exp \left(-\sqrt{\xi}-\frac{1}{2} \square\right)\right|_{T},\right.
$$

where straight lines denote gauge transporters (including the static propagators) and wiggly lines stand for light quark propagators. The $Q Q q$ potentials only depend on the distance and a $D_{\infty h}^{\prime}$ representation (see below). The light quark and gluon degrees of freedom have been integrated out. 
One can use the resulting potentials as an input to a Schrödinger equation, in the heavy quark limit, and apply the formalism of NRQCD for corrections.

\section{Simulation details}

We use 68 QCDSF configurations [4]. The specifications are displayed in table 1. We use

\begin{tabular}{|l|l|l|l|l|l|l|l|}
\hline Lattice points & $\kappa_{\text {val }}$ & $\kappa_{\text {sea }}$ & $\beta$ & $C_{S W}$ & $m_{\pi}[\mathrm{GeV}]$ & $a[\mathrm{fm}]$ & $L[\mathrm{fm}]$ \\
\hline $16^{3} \times 32$ & 0.1355 & 0.1355 & 5.29 & 1.9192 & $0.783(11)$ & $0.084(1)$ & 1.3 \\
\hline
\end{tabular}

Table 1: Wilson-Clover $N_{f}=2$ lattices.

all-to-all propagators with 300 stochastic estimates on each configuration. We apply the hopping parameter expansion [5, 6] to reduce the stochastic noise. APE-smeared [7, 8] gauge links were used for the spatial gauge connectors in the Wilsonloops and the link covariant displacements. For the static quark propagator we use temporal links that have been stout smeared [9] once, to reduce the static energy and thus to improve the signal to noise ratio. The quark fields of the all-to-all propagators are Wuppertal smeared [10] using APE-smeared gauge transporters and we applied the variational method $[11,12]$ to extract the groundstate (GS), the first excitation and second excitations (FE, SE). With this setting we follow the methods described in [6]. We compute the baryon correlator with two static quarks in various geometries. The light quark can be in the middle which we will refer to as $\square$,

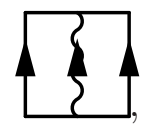

or the light quark can be placed at one of the static quark positions, symmetric $(\oplus)$ or antisymmetric $(\ominus)$,

$$
(4 \sqrt[3]{5} \hat{3}) \pm(\sqrt[3]{5}+\sqrt{5}) .
$$

All these correlators are realized by using the appropriate gauge connectors $\mathscr{P}$ in the baryon interpolator

$$
O\left(x, x^{\prime}, x^{\prime \prime}\right)^{\alpha}=\varepsilon_{a b c} P_{+}^{\alpha \alpha^{\prime}} Q(x)_{a^{\prime}}^{\alpha^{\prime}} \mathscr{P}\left(x, x^{\prime \prime}\right)_{a^{\prime} a}\left(Q\left(x^{\prime}\right)_{b^{\prime}}^{\beta} \mathscr{P}\left(x^{\prime}, x^{\prime \prime}\right)_{b^{\prime} b} \Gamma^{\beta \gamma} q\left(x^{\prime \prime}\right)_{c}^{\gamma}\right),
$$

where all coordinates $x$ are at the same time $t, P_{+}=\frac{1}{2}\left(\mathbb{1}+\gamma_{4}\right)$ is the projector to positive parity and $\Gamma$ is one of the operators from table 2. We denote the separation of the two heavy quarks by $r$. We use the same operator $\Gamma$ for source and sink so that the correlator we compute is characterized by $r, \Gamma$, the temporal extent of the correlator $T$ and the geometry $(\square, \oplus, \ominus$ ). For $r=0$, $\square$ and $\oplus$ are degenerate.

The octahedral group $O_{h}$ is the cubic group of rotations on the lattice, with the addition of parity. Its irreducible representations describe point-particles at rest on a discrete lattice. The mapping between these and the continuum quantum numbers is not unique and so an lattice irreducible representation can correspond to multiple continuum $J$ states. 
In our case, at $r>0$, this symmetry is broken to the cylindrical subgroup $D_{4 h} \subset O_{h}$. In the continuum this corresponds to $D_{\infty h} \subset O(3)$. As we are interested in baryons we have to take a spinorial irreducible representation (irrep). We refer to these representations with half integer values of $J$ as $O_{h}^{\prime}$ or $D_{\infty h}^{\prime} / D_{4 h}^{\prime}$. In table 2 we list the operators that we have used to calculate different correlators

\begin{tabular}{|c|c|c|c|}
\hline & & $r=0$ & $r>0$ \\
\hline Wave & Operator & $O^{\prime}(3), O_{h}^{\prime}$ & $D_{\infty h}^{\prime}, D_{4 h}^{\prime}$ \\
\hline$S$ & $\gamma_{5}$ & $\frac{1}{2}^{+}, G_{1}^{+}$ & $\frac{1}{2}, G_{1 g}$ \\
\hline$P_{-}$ & $\mathbb{1}$ & $\frac{1}{2}^{-}, G_{1}^{-}$ & $\frac{1^{2}}{2}, G_{1 u}$ \\
\hline$P_{+}$ & $\gamma_{1} \Delta_{1}-\gamma_{2} \Delta_{2} \oplus$ cyclic & $\frac{3}{2}^{-}, H^{-}$ & $\begin{array}{l}\frac{3}{2} u, G_{2 u} \\
\frac{1}{2}{ }_{u} \perp, G_{1 u}\end{array}$ \\
\hline$D_{-}$ & $\gamma_{5}\left(\gamma_{1} \Delta_{1}-\gamma_{2} \Delta_{2}\right) \oplus$ cyclic & $\frac{3}{2}^{+}, H^{+}$ & $\begin{array}{l}\frac{3}{2} g \|, G_{2 g} \\
\frac{1}{2}{ }_{g} \perp, G_{1 g}\end{array}$ \\
\hline$D_{+}$ & $\gamma_{1} \Delta_{2} \Delta_{3}+\gamma_{2} \Delta_{3} \Delta_{1}+\gamma_{3} \Delta_{1} \Delta_{2}$ & $\frac{5}{2}^{+}, G_{2}^{+}$ & $\frac{1}{2}, G_{1 g}$ \\
\hline$F_{-}$ & $\gamma_{5}\left(\gamma_{1} \Delta_{2} \Delta_{3}+\gamma_{2} \Delta_{3} \Delta_{1}+\gamma_{3} \Delta_{1} \Delta_{2}\right)$ & $\frac{5}{2}^{-}, G_{2}^{-}$ & $\frac{1}{2} u / \frac{5}{2} u, G_{1 u}$ \\
\hline
\end{tabular}

Table 2: List of operators and representations.

and their corresponding quantum numbers. We denote the link covariant displacement as,

$$
\Delta_{\mu} \eta(x)=U_{\mu}(x) \eta(x+a \mu)-U_{-\mu}(x) \eta(x-a \mu) \quad \text { where } \quad U_{-\mu}(x)=U_{\mu}^{\dagger}(x-\mu) .
$$

We have the correspondence

$$
G_{1} \leftarrow \frac{1}{2}, \frac{5}{2}, \cdots \quad \text { and } \quad G_{2} \leftarrow \frac{3}{2}, \frac{7}{2}, \cdots
$$

between the $D_{4 h}^{\prime}$ lattice and the $D_{\infty h}^{\prime}$ continuum quantum numbers. For $r>0$ some $O_{h}^{\prime} / O(3)^{\prime}$ irreps will split up into two or more $D_{4 h}^{\prime} / D_{\infty h}^{\prime}$ irreps. For instance, the operators corresponding to $H^{ \pm}$split up, depending on the direction, relative to the interquark axis: for the axis pointing into the $\hat{3}$-direction, we call the operator $\gamma_{1} \Delta_{1}-\gamma_{2} \Delta_{2}$ "parallel" $(\|)$ and the other combinations "perpendicular" $(\perp)$, where $\perp$ corresponds to the state of lower angular momentum $\left(G_{1} / \frac{1}{2}\right)$ and $\|$ to the higher angular momentum $\left(G_{2} / \frac{3}{2}\right)$, relative to the $\hat{3}$-axis.

\section{Results}

\subsection{Spectrum}

The spectrum of our observables at $r=0$ is shown in figure 2(a). The Regge trajectories are denoted by the grey lines and although they are not examined further they help to group the operators. The spectrum extends over a range of $2 \mathrm{GeV}$ and it gets even richer when one goes to $r>0$ where the symmetry group $O_{h}^{\prime}$ breaks down into $D_{4 h}^{\prime}$.

The states created by the operators $P$ and $P \gamma_{5}$ split up into two energy levels depending on whether or not the angular momentum contains a projection onto the $Q Q$ axis. The spectrum at $r=5 a$ is shown in figure 2(b) and the grey lines in the plot show degeneracies of some of the 


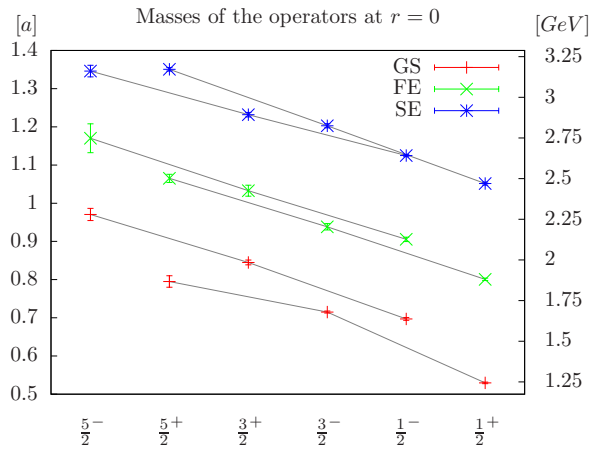

(a) $r=0$

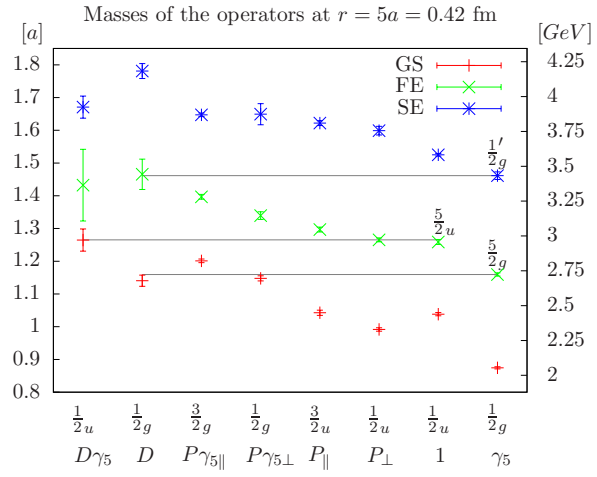

(b) $r=5 a$

Figure 2: The different operators at $r=0$ and $r=5 a$, with the lowest possible continuum spin assignments.

levels. For example, the excitation of $\mathbb{1}$ and the ground state of $D \gamma_{5}$ are degenerate. Both are in the $G_{1 u}$ representation of $D_{4 h}^{\prime}$, corresponding to the continuum $D_{\infty h}^{\prime}$ quantum numbers $\frac{1}{2}{ }_{u}, \frac{5}{2}{ }_{u}, \ldots$ The latter operator corresponds to the $O(3)$ quantum numbers $\frac{5}{2}^{-}$at $r=0$. Hence we assign a continuum $\frac{5}{2}{ }_{u}$ spin to it and to the first radial excitation of the 1 operator. Unfortunately the two ground states of $\frac{1}{2} u$, i.e. $\mathbb{1}$ and $P \perp$ seem to be different and it looks like $\frac{1}{2} u \simeq \mathbb{1}$ is much closer to $\frac{3}{2}{ }_{u} \simeq P \|$. We hope to resolve this issue in the near future.

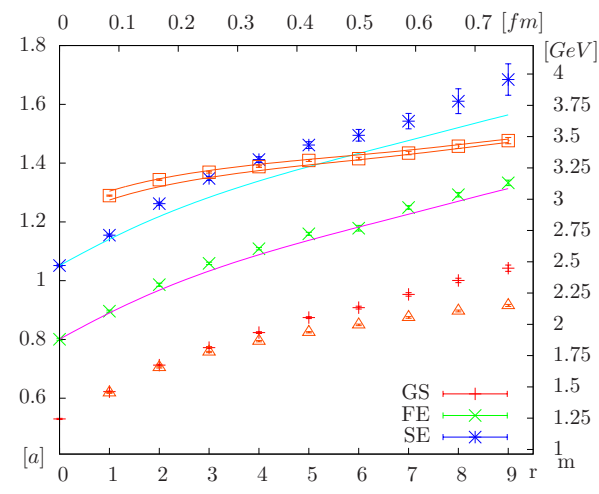

Figure 3: Potential for $\frac{1}{2}_{g} \simeq \gamma_{5} \oplus$.

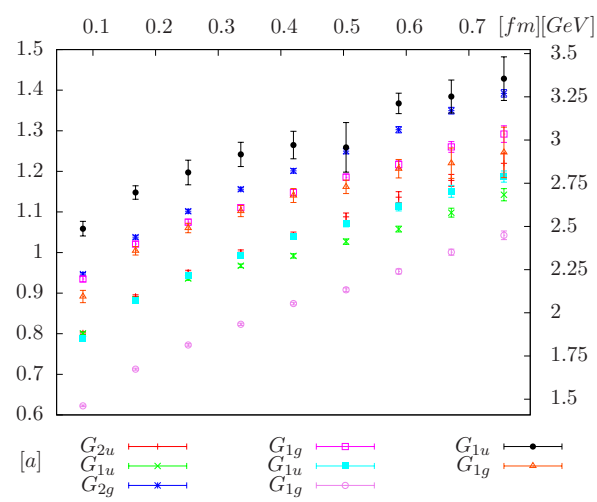

Figure 4: Lowest lying potential for each operator.

\subsection{Potentials}

In figure 3 we plot the results of $\frac{1}{2}_{g} \simeq \gamma_{5} \oplus$ as red, green and blue symbols. The factorization model expectation for the ground state $m_{\bar{Q} q}+\frac{1}{2} V_{Q \bar{Q}}$ is represented by the orange triangles. For $r>3 a \approx 0.25 \mathrm{fm}$ the data lie systematically above the expectation. The increase in energy with increasing $r$ originates from the internal energy of the $Q Q$ diquark which, in the presence of the light quark, rises more steeply then expected. In figure 4 we see that this slope depends on the $D_{4 h}^{\prime}$ quantum numbers, such that this failure of the factorization ansatz cannot be attributed to a different functional form of the $Q Q$ potential alone. 
The pink and light blue lines are interpolations of the ground state points, shifted by the first two static-light energy splittings. The pink line describes the first excitation of the baryonic potential very well. This suggests that this excitation is due to the light quark, with very little effect from the gluonic flux configuration. The second excited state data lie somewhat above the light blue line. The most likely reason for this deviation is our inability to reliably isolate this excitation in our three-dimensional variational basis, so that we somewhat overestimate the masses.

At large $r$ one of the static quarks might form a diquark with the light quark. In this case we should see the transverse modes of the string connecting this $Q q$ diquark with the remaining $Q$ in the excitation spectrum. The Nambu-Goto string potential [13] suggests the following functional form:

$$
E_{n}(r)=\sigma_{\mathrm{GS}} r \sqrt{1+\left(2 n-\frac{d-2}{12}\right) \frac{\pi}{\sigma_{\mathrm{GS}} r^{2}}} .
$$

In our case $d=4$ and $\sigma_{\mathrm{GS}}$ is the effective string tension of the ground state determined by fitting to

$$
V_{Q Q q}(r)=C_{\text {eff }}+\sigma_{\text {eff }} r-\frac{A_{\text {eff }}}{r} .
$$

The red band is $E_{2}-E_{0}+\mathrm{GS}$, which is the first candidate for an excitation because $n=1$ would affect the spin of the system. This curve is above our first radial excitation and its shape is very different from the second excitation. The light quark excitation energies are smaller than those required for transverse excitations of the flux tube. The plots for the other observables look very similar.

\subsection{Wavefunctions}

We compute the RMS and the wavefunctions of the ground state and the first two excitations as outlined in [14]. The light quark RMS for the ground state wavefunction created by the operator $\gamma_{5}$ is about 4.1a. Within errors it is independent of the geometry of the correlator. This distance is indeed reasonably close to $r \approx 0.3 \mathrm{fm}$, where the factorization ansatz starts to fail. The shapes of the Coulomb gauge wavefunctions agree with the findings of [14] although this reference considered mesons while we study the light quark distribution inside a $Q Q q$-baryon.

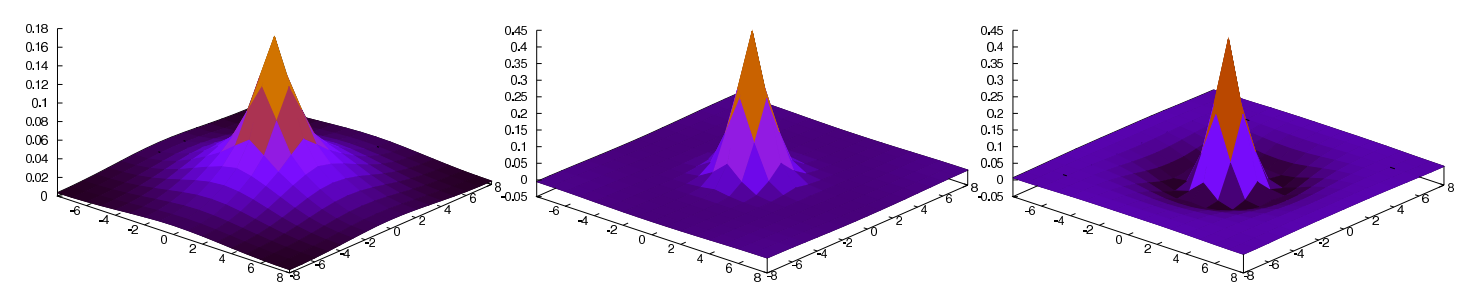

Figure 5: Wavefunction of groundstate and first two excitations of $\gamma_{5} \oplus$ for $r=4$.

\section{Conclusion and Outlook}

The factorization model breaks down for separations greater than $0.3 \mathrm{fm}$. The nature of the first excitation of a static-static-light baryon does not change with an increase of the separation in 
the two static quarks. The second excitation could not be resolved so well and we do not venture to explain its nature. The splittings induced by breaking the symmetry group $O_{h}$ into $D_{4 h}$ are reflected in our correlators.

It is a puzzle why the states of $\frac{1}{2} u$ coming from the operators $\mathbb{1}$ and from $P_{\perp}$ do not agree but instead the $\mathbb{1}$ seems to be degenerate with the $\frac{3}{2} u$ from $P_{\|}$. At the moment we are conducting a study on a bigger lattice $\left(24^{3} \times 48\right)$ at smaller quark masses $(\kappa=0.1362)$ and we hope to clarify this point.

\section{Acknowledgments}

We thank the QCDSF Collaboration for making their configurations available on the ILDG. We use the chroma package [15] for our simulations. This work was supported by the DFG Sonderforschungsbereich/Transregio 55 and BMBF grant 06RY257.

\section{References}

[1] A. Yamamoto, H. Suganuma and H. Iida, Lattice QCD study of the heavy-heavy-light quark potential., Phys. Rev. D78 (2008) 014513. [arXiv:0806.3554 [hep-lat]]

[2] M. J. White and M. J. Savage, Semileptonic decay of baryons with two heavy quarks., Phys. Lett. B271 (1991) 410.

[3] M. J. Savage and M. B. Wise, Spectrum of baryons with two heavy quarks., Phys. Lett. B248 (1990) 177.

[4] D. Brommel et al., The pion form factor from lattice QCD with two dynamical flavours, Eur. Phys. J. C51 (2007) 335 [hep-lat/0608021].

[5] C. Thron, S. J. Dong, K. F. Liu, and H. P. Ying. Pade-Z(2) estimator of determinants., Phys. Rev. D57 (1998) 1642. [hep-lat/9707001]

[6] G. S. Bali, H. Neff, T. Düssel, T. Lippert and K. Schilling, Observation of string breaking in QCD, Phys. Rev. D71 (2005) 114513 [hep-lat/0505012].

[7] M. Albanese et al. Glueball Masses and string tension in Lattice QCD., Phys.Lett. B192 (1987) 163.

[8] M. Teper An improved method for lattice glueball calculations., Phys. Lett. B183 (1987) 345.

[9] C. Morningstar and M. J. Peardon, Analytic smearing of SU(3) link variables in lattice QCD., Phys. Rev. D69 (2004) 054501. [hep-lat/0311018]

[10] S. Güsken et al. Non-singlet axial vector couplings of the baryon octet in lattice QCD., Phys. Lett. B227 (1989) 266.

[11] M. Lüscher and U. Wolff. How to calculate the elastic scattering matrix in two-dimensional quantum field theories by numerical simulation., Nucl. Phys. B339 (1990) 222.

[12] C. Michael, Adjoint sources in Lattice Gauge Theory., Nucl. Phys. B259 (1985) 58.

[13] J. F. Arvis, The exact q $\bar{q}$ potential in Nambu string theory, Phys. Lett. B127 (1983) 106.

[14] C. Ehmann, Charmonium spectrum including higher spin and exotic states, PoS (LATTICE2007) (2007) 094 [arXiv:0710.0256 [hep-lat]].

[15] R. G. Edwards and B. Joo, The Chroma Software System for Lattice QCD, Nucl.Phys.Proc.Suppl. (2005) 140. [hep-lat/0409003] 